\title{
Effect of Modifying Agent (ACR) on Cell Structure and Mechanical Properties of Extrusion-Foamed PVC Sheet
}

\author{
Mingyi Wang ${ }^{1, a}$, Nanqiao Zhou ${ }^{2, b}$ and Jun $\mathrm{Hu}^{2, \mathrm{c}}$ \\ ${ }^{1}$ School of Mechanical and Power Engineering, East China University of Science and Technology, \\ Shanghai 200237, China \\ ${ }^{2}$ Key Laboratory of Polymer Processing Engineering, Ministry of Education, National Engineering \\ Research Center of Novel Equipment for Polymer Processing, South China University of Technology, \\ Guangzhou 510640, China

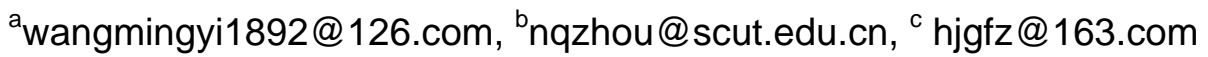

Keywords: PVC; foam; extrusion; supercritical carbon dioxide

Abstract. Rigid polyvinyl chloride (R-PVC) foam sheets were prepared in a continuous extrusion foaming system by using supercritical $\mathrm{CO}_{2}$ as the foaming agent. The effects of acrylic resin (ACR) on the rheological properties of PVC were investigated using a Brabender torque rheometer while other basic formula remained unchanged. The influences of ACR content on microstructure, and mechanical properties of PVC foamed sheet were investigated. The rheological test results showed that with the addition of ACR, the gelation time of the formula was gradually shortened while the equilibrium torque increased. With the increase of ACR content in the formula, the tensile strength, elongation at break, flexural strength and notched impact strength of PVC increased initially and then decreased, implying that there exists an optimum ACR content for improving the mechanical properties of foamed PVC sheet.

\section{Introduction}

Rigid PVC foam is in growing demand due to its application in various fields as a substitute for wood [1]. Besides many advantages similar to natural wood, R-PVC foam also has many merits like fire resistance, low moisture absorption, low thermal conductivity, strong ability to resist pest attack, together with superior heat and sound insulation [2]. ACR is commonly used as the impact modifier to improve PVC's rheological properties, promote melting and improve melt elasticity [3-5].

To the best of my knowledge, there is no research systematically conducted on the effects of ACR content on microstructure, tensile strength, flexural strength and notched impact strength of PVC foam sheet by using supercritical $\mathrm{CO}_{2}$ as the foaming agent. This study was made in order to find out the optimum content of ACR as the modifying agent in extrusion foaming of PVC with supercritical $\mathrm{CO}_{2}$ as the foaming agent. The influences of ACR content on microstructure and mechanical properties of foamed PVC sheet were studied in detail with the help of scanning electron microscopy (SEM) and the corresponding mechanical properties tests.

\section{Experimental}

\section{Materials}

The polymer matrix used in this study was PVC (Tianjin Dagu Chemical Co., Ltd, ZS-85). Organotin (TS181) and stearic acid (HST) were used as the heat stabilizer. Ester lubricant (P30) and polyethylene wax were used as the in-lubricant and out-lubricant respectively. In addition, acrylic resin (ACR, Jiangxi Yuefeng macromolecule material Co., Ltd., YFG-530) and DOP were used as the modifying agent and plasticizer. Active calcium carbonate $\left(\mathrm{CaCO}_{3}\right.$, Guangdong Yan Hua Chemical Industry Co., Ltd, CCR) was used as the nucleating agent. Supercritical $\mathrm{CO}_{2}$ (Gold pearl river Co., Ltd. of Guangzhou, commercial 99\% min) was utilized as the foaming agent. All chemicals received from the manufactures remained unmodified. 
The dry blending of PVC and other additives was carried out in a high speed mixer until obtaining a homogeneous mixture. The concentrations of each ingredient in the formula of the composites are listed in Table 1. The ACR concentration changed from 5phr to 8phr to check its effect on rheological properties of the PVC formula and microstructure, as well as mechanical properties of the foamed PVC sheet obtained. After dry blending, the compounded mixtures were extruded through a twin-screw extruder to prepare pellets for extrusion foaming. Extrusion foaming experiments were conducted on a $45 \mathrm{~mm}$ single-screw extruder foaming system designed by ourselves with L/D ratio of 29:1. The width and thickness of the sheet die were $80 \mathrm{~mm}$ and $2 \mathrm{~mm}$ respectively. For extrusion foaming, the screw speed was 7rpm, as a result of which the MFR of the extrudate from the sheet die was kept at about $57.5 \mathrm{~g} / \mathrm{min}$, and the injection rate of supercritical $\mathrm{CO}_{2}$ was maintained at about $2 \mathrm{ml} / \mathrm{min}$. The temperature profile from hopper to sheet die was set at $160 / 170 / 175 / 170 / 165^{\circ} \mathrm{C}$. From foamed-extrudates, dogbone-shaped specimens with a dimension of $25 \times 4 \times 3 \mathrm{~mm}$ were prepared for the following tensile test.

Table 1 Formulas used for PVC

\begin{tabular}{lc}
\hline Material & Content [phr] \\
\hline PVC & 100 \\
Organotin stabilizer & 2 \\
PE wax & 1 \\
Ester lubricant & 3 \\
Stearic acid & 0.3 \\
$\mathrm{CaCO}_{3}$ & 10 \\
DOP & 2 \\
$\mathrm{ACR}$ & $5,6,7,8$ \\
\hline
\end{tabular}

\section{Foam Characterization}

The density of the foamed samples was determined by the buoyancy method. For details of the method, see reference [6]. SEM was used to characterize cell morphology of the foamed samples. All of the foamed samples were dipped into liquid nitrogen and then quickly fractured in air before scanning. Then the fractured cross section of the samples was sputter-coated with platinum in an E-1010 mini sputter coater.

\section{Mechanical Properties Testing}

Tensile testing was performed on a universal testing machine with a crosshead displacement rate of $1 \mathrm{~mm} / \mathrm{min}$. The tensile strength and elongation at break of the foam sample were calculated using the formula (1) and (2):

$$
\begin{aligned}
& \sigma_{\mathrm{t}}=\frac{F}{\mathrm{bd}}(M P \mathrm{a}) \\
& \varepsilon_{\mathrm{t}}=\frac{L-L_{0}}{L_{0}}
\end{aligned}
$$

Where F represents the maximum tensile load of the samples $(\mathrm{N})$, $\mathrm{b}$ and $\mathrm{d}$ represents the width $(\mathrm{mm})$ and thickness $(\mathrm{mm})$ of the samples tested. $\mathrm{L}_{0}$ is the initial value of the gage part of the standard sample $(\mathrm{mm}) ; \mathrm{L}$ is final value of the gage part of the standard sample at break $(\mathrm{mm})$. The flexural properties of the foamed samples were conducted on a universal testing machine according to standard GB/T 9340-2000 while notched impact strength of the foamed samples was performed on a pendulum impact testing machine according to standard GB/T 1843-1996.

\section{Effect of ACR content on the rheological properties of PVC formula system}

Four different formulas in which ACR content ranges from 5 to 8 phr were used in our experiment to investigate the effect of ACR content on the rheological properties of PVC formula system on the premise that other components of the formulas were invariable (see Table 1). Fig. 1 shows the rheological curve of the PVC formula system with different ACR dosages when the rotational speed of the torque rheometer was $30 \mathrm{r} / \mathrm{min}$ and processing temperature was $175^{\circ} \mathrm{C}$. As can be seen in Fig. 1 , 
the gelation time of the formula was gradually shortened while the equilibrium torque increased. The potential mechanism might be as follows: ACR can disperse in PVC melt into reticular structure units of less than 0.01 microns, and evenly distributed in PVC particles. Under shearing action, the reticular structure units can increase the friction between PVC particles and increase molecular entanglement, promoting the heat and mass transfer in PVC melt and shortening the melting time of PVC particles.

\section{Effect of ACR content on microstructure of foamed PVC sheet}

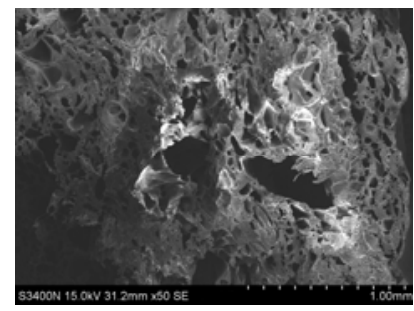

(a) $5 \mathrm{phr}$

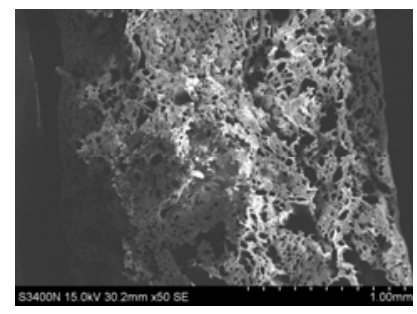

(b) $6 \mathrm{phr}$

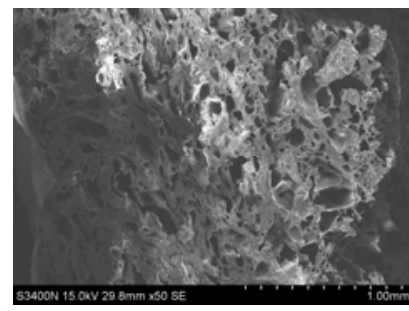

(c) $7 \mathrm{phr}$

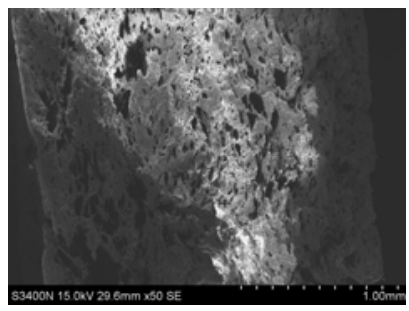

(d) $8 \mathrm{phr}$

Fig.1 Micrographs of cross section of foamed PVC sheet with different concentrations of ACR

In Fig.1, SEM micrographs of foamed PVC sheet with different concentrations of ACR are shown. It can be seen that with the increased concentration of ACR, bubble coalescence gradually reduced. When 5phr and $6 \mathrm{phr}$ ACR was included in the formula, bubble coalescence phenomenon was obvious and some large and oblate bubbles appeared, as shown in Fig. 1(a) and Fig. 1(b). By comparison, the cell morphology of foamed PVC sheet in Fig. 1(c) is relatively uniform, when 7 phr ACR was added to the formula. As ACR concentration in the PVC formula was further increased to 8 phr, the number of cells in the foamed PVC sheet decreased a lot, which means that excessive addition of DOP in the formula is not good for foaming of PVC.

\section{Effect of ACR content on tensile properties of foamed PVC sheet}

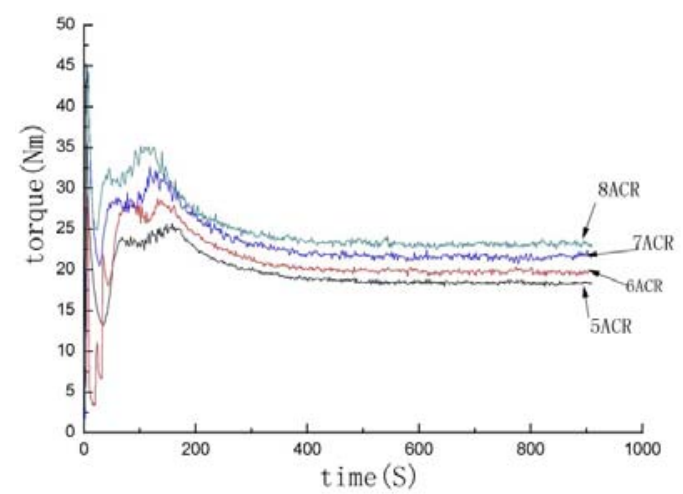

Fig.2 Rheological curve of the PVC formula system with different ACR contents

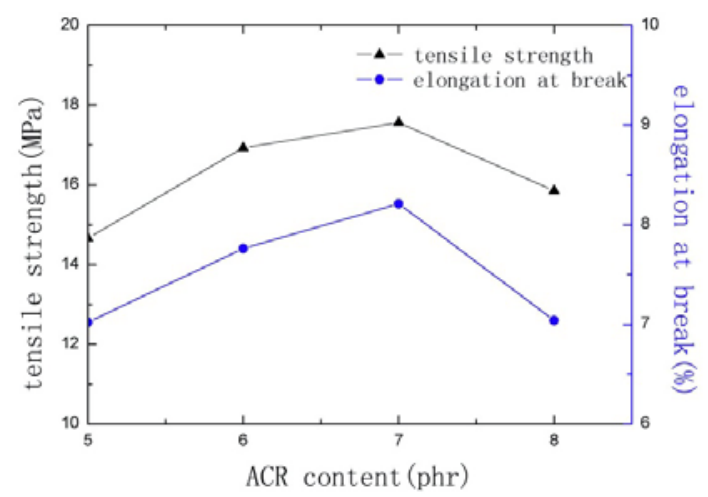

Fig.3 Tensile properties of foamed PVC sheets with different concentrations of ACR

As can be seen from Fig.3, both of the tensile strength and the elongation at break of the foamed PVC samples first increased as ACR content was increased from $5 \mathrm{phr}$ to $7 \mathrm{phr}$ and then decreased as ACR content was further increased to 8 phr. This is because that the addition of ACR can improve the melt strength of PVC, which can inhibit bubble growth and prevent bubble coalescence in the stage of bubble growth, resulting in increased cell-population density and reduced cell size, which is beneficial to improvement of tensile properties of foamed PVC sheets. However, as ACR content in PVC formula was further increased to 8 phr, the melt strength of PVC might become too high, which 
suppressed the foaming process, resulting in foamed PVC sheet with uneven bubbles and reduced tensile properties.

\section{Effect of ACR content on flexural strength and notched impact strength of foamed PVC sheet}

Fig.4 shows the effect of ACR content on flexural strength of foamed PVC sheet. It is shown that the flexural strength of the foamed PVC sheet first increased as ACR content was increased from 5 phr to $7 \mathrm{phr}$ and then reduced as ACR content was further increased to $8 \mathrm{phr}$. The maximum flexural strength was reached when $7 \mathrm{phr}$ ACR was incorporated in PVC formula. The underlying mechanism was that the addition of ACR increased the melt strength, resulting in foamed PVC sheet with fine cell structure, which improved the flexural strength of PVC foam. However, as 8 phr ACR was added to formula, excessive addition of ACR caused too high melt strength, which suppressed the foaming process, resulting in foams with uneven cell morphology, which made the flexural strength of the PVC foam decrease.

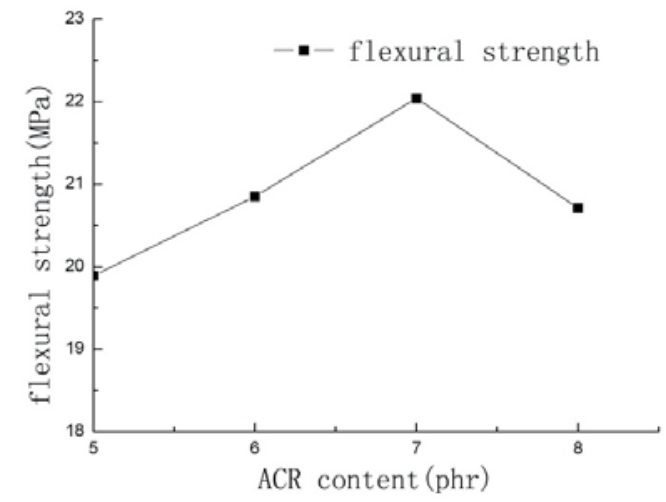

Fig.4 Effect of ACR content on flexural strength of foamed PVC sheet

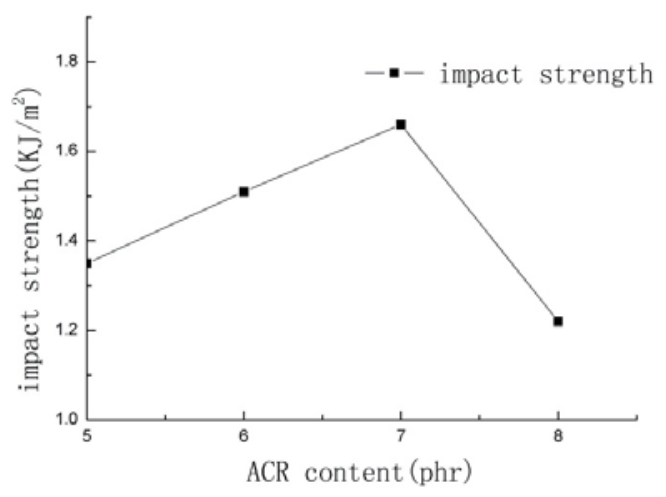

Fig.5 Effect of ACR content on notched impact strength of foamed PVC sheet

Fig.5 illustrates the effect of ACR content on notched impact strength of foamed PVC sheet, it can be seen that the notched impact strength first increased as ACR content was increased from 5 phr to 7 phr and then decreased as ACR content was further increased to 8 phr. Foamed PVC sheet with the maximum notched impact strength was reached when 7 phr ACR was incorporated in PVC formula.

\section{Conclusions}

Through the study of effects of ACR content on rheological property of PVC formula system and foam structure, as well as mechanical properties of the obtained foamed PVC sheets, the following conclusions were drawn:

1. The gelation time of the formula was gradually shortened while the equilibrium torque increased with the increase of ACR concentration in PVC formula.

2. Foamed PVC sheet with the maximum tensile strength, elongation at break, flexural strength and notched impact strength was reached when 7 phr ACR was incorporated in PVC fomulation.

3. Foamed PVC sheet with relatively uniform cell structure was obtained when 7 phr ACR was added to the formula.

\section{Acknowledgements}

This work was supported by the National Natural Science Foundation of China (Grant No. 21206152).

\section{References}

[1] B. Azimipour, F. Marchand. Journal of Vinyl \& Additive Technology, Vol. 12(2006), p. 55 
[2] J. Patterson. Journal of Vinyl \& Additive Technology, Vol. 7(2001), p. 138

[3]L.M. Matuana, F. Mengeloglu. Journal of Vinyl \& Additive Technology, Vol. 7(2001), p. 67

[4]H. Chang, N.A. Zahra. Journal of Vinyl \& Additive Technology, Vol. 17(2011), p. 17

[5] M.Y. Wang, N.Q. Zhou, S.P. Wen. Polymer - Plastics Technology and Engineering, Vol. 48(2009), p. 303

[6]M.Y. Wang, J. Ma, R. Chu, C.B. Park, N.Q. Zhou. Journal of Applied Polymer Science, Vol. 123(2012), p. 2726 